\title{
Indication for Co-evolution of Lactobacillus johnsonii with its hosts
}

\author{
Keren Buhnik-Rosenblau', Vera Matsko-Efimov' ${ }^{1}$ Minju Jung ${ }^{2}$, Heuynkil Shin², Yael Danin-Poleg ${ }^{1}$ \\ and Yechezkel Kashi ${ }^{1 *}$
}

\begin{abstract}
Background: The intestinal microbiota, composed of complex bacterial populations, is host-specific and affected by environmental factors as well as host genetics. One important bacterial group is the lactic acid bacteria (LAB), which include many health-promoting strains. Here, we studied the genetic variation within a potentially probiotic LAB species, Lactobacillus johnsonii, isolated from various hosts.
\end{abstract}

Results: A wide survey of 104 fecal samples was carried out for the isolation of L. johnsonii. As part of the isolation procedure, terminal restriction fragment length polymorphism (tRFLP) was performed to identify L. johnsonii within a selected narrow spectrum of fecal LAB. The tRFLP results showed host specificity of two bacterial species, the Enterococcus faecium species cluster and Lactobacillus intestinalis, to different host taxonomic groups while the appearance of $L$. johnsonii and $E$. faecalis was not correlated with any taxonomic group. The survey ultimately resulted in the isolation of $L$. johnsonii from few host species. The genetic variation among the $47 \mathrm{~L}$. johnsonii strains isolated from the various hosts was analyzed based on variation at simple sequence repeats (SSR) loci and multi-locus sequence typing (MLST) of conserved hypothetical genes. The genetic relationships among the strains inferred by each of the methods were similar, revealing three different clusters of $L$. johnsonii strains, each cluster consisting of strains from a different host, i.e. chickens, humans or mice.

Conclusions: Our typing results support phylogenetic separation of L. johnsonii strains isolated from different animal hosts, suggesting specificity of $L$. johnsonii strains to their hosts. Taken together with the tRFLP results, that indicated the association of specific LAB species with the host taxonomy, our study supports co-evolution of the host and its intestinal lactic acid bacteria.

\section{Background}

The intestinal microbiota consists of hundreds to thousands of bacterial species which play an important role in normal gut functioning and are crucial for maintaining the organism in good health. It is composed of complex bacterial populations that have recently been found to be host-specific [1-3], a result of variations in environmental factors [4-6] and host genetics [7-11].

One important group of bacteria colonizing the gut is the lactic acid bacteria (LAB), a heterogeneous group of gram-positive rods and cocci that belong to the phylum Firmicutes. There are indications of a correlation between oral administration of some LAB strains and

\footnotetext{
* Correspondence: kashi@tx.technion.ac.il

'Department of Biotechnology and Food Engineering, Technion-Israel Institute of Technology, Haifa, Israel

Full list of author information is available at the end of the article
}

improvement of gut health disorders, such as pouchitis, ulcerative colitis, infectious diarrhea, antibiotic-associated diarrhea, traveler's diarrhea, necrotizing enterocolitis, atopic eczema and Helicobacter pylori infections [12-16]. The largest bacterial genus in the LAB is Lactobacillus. It is highly diverged and consists of over a hundred species $[17,18]$. Lactobacilli are widely used in food fermentation and are well known for their preservative ability as well as for their positive contribution to texture and flavor formation in many food products. In addition, several well-characterized probiotic strains (live microorganisms which, when administered in adequate amounts, confer a health benefit on the host; FAO/ WHO Guidelines, 2002, ftp://ftp.fao.org/es/esn/food/ wgreport2.pdf) belonging to this genus are used by the food and pharmaceutical industries, and new probiotic lactobacilli strains are discovered. One of the most

\section{() Biomed Central}


intensively investigated Lactobacillus species is Lactobacillus johnsonii, which has been reported so far to inhabit the gastrointestinal tracts (GITs) of several hosts, including humans, mice, dogs, poultry, pigs and honeybees [1923]. Specific $L$. johnsonii strains are known for their probiotic activities [24-28] and some, such as L. johnsonii NCC 533 [29], are also used by the industry. Probiotic characteristics are presented by various $L$. johnsonii strains, including inhibition of different pathogens in the chick gut, alleviation of diabetes symptoms, reduction of serum cholesterol levels, immunostimulation and adherence to intestinal epithelial cells [24,26-29].

Due to increased interest in $L$. johnsonii, various molecular tools have been used for the precise differentiation of L. johnsonii from other members of the Lactobacillus acidophilus cluster, particularly the closely related species Lactobacillus gasseri [30-33]. The fact that different strains display different characteristics highlights the need to develop tools for their accurate discrimination as well. Various methods have been recently used to type L. johnsonii strains, such as pulsed field gel electrophoresis, amplified fragment length polymorphism, enterobacterial repetitive intergenic consensus PCR and repetitive extragenic palindromic PCR $[20,21,33$,$] . These typing methods differ in their discrim-$ inatory power, rapidity, complexity, cost, reliability and reproducibility.

In this study we used simple sequence repeats (SSR), also termed variable number tandem repeats (VNTR). SSR loci presents inherently high mutation rate [34], which makes them an appropriate tool for strain typing in many bacterial species [35-37].

Another bacterial typing method based on sequence variations is multiple locus sequence typing (MLST) [38], mainly of housekeeping genes, providing an indication of relatively distant evolutionary processes [39]. Similarly, conserved hypothetical genes can provide an additional source of sequence variation [40]. This cluster of genes with unknown function is predicted to be present in the genomes of all members of a particular species.

In this study L. johnsonii was identified and isolated from a selected narrow spectrum of the fecal LAB population originated from various animal hosts. The genetic relationships among $L$. johnsonii strains were inferred based on variation at selected sets of SSR loci and MLST of conserved hypothetical genes. Our findings suggest specificity of L. johnsonii strains to their hosts.

\section{Results}

Isolation of $L$. johnsonii from various animal hosts and characterization of their selected fecal LAB populations

A large survey for L. johnsonii isolation was performed, where 104 fecal samples originating in six host taxonomic classes were tested. The isolation procedure of
L. johnsonii relied on few methods: identifying $L$. johnsonii within a narrow spectrum of fecal LAB populations using terminal restriction fragment length polymorphism (tRFLP) analysis and isolation of suspected L. johnsonii colonies based on their morphology followed by species-specific PCR amplification of $23 S$ rDNA and $16 \mathrm{~S}$ rDNA sequencing.

The tRFLP patterns of the fecal LAB populations grown on the selective medium $\mathrm{mEnterococcus} \mathrm{agar}$ (based on [8]) generally presented four major peaks representing the Enterococcus faecium species cluster, Lactobacillus intestinalis, L. johnsonii, and Enterococcus faecalis. We compared the relative abundance of these bacterial species obtained from 50 different animal hosts from a wide variety of taxonomic groups (Figure 1). No correlation was found between the relative abundance of any of the four bacterial species and either geographical location or nutritional habits of the host (data not shown). However, a correlation was found between the taxonomy classification of the host and the relative abundance of two bacterial species: L. intestinalis and the E. faecium species cluster. The E. faecium cluster was highly abundant in most samples (Figure 1), but appeared at significantly lower levels in samples originated from hosts belonging to the Rodentia ( $\mathrm{F}=32.3975$, $p<0.0001)$. In contrast, $L$. intestinalis was relatively rare in general, but was significantly more abundant in samples originated from hosts belonging to the Rodentia $(\mathrm{F}=6.5525, p=0.0133)$. In addition, the presence of $L$. intestinalis was correlated with the absence of $E$. faecium cluster and vice versa. In contrast to the E. faecium cluster and L. intestinalis, the appearance of $L$. johnsonii and $E$. faecalis did not correlate with any taxonomic group. In a second step we studied L. johnsonii to the strain level. All together 39 strains were isolated from few tested animal hosts (Table 1, Additional file 1: Origin of samples collected from 104 animal hosts) and further genetically characterized.

\section{Polymorphism at SSR loci along the $L$. johnsonii genome}

In silico genome-wide screen of L. johnsonii NCC 533

revealed thousands of SSR tracts that were evenly distributed and highly abundant along the genome

Eleven loci with the largest number of repeats were chosen for genetic characterization of L. johnsonii (Table 2), having motif sizes ranging from 1 to $480 \mathrm{bp}$. Ten SSR loci were located in coding regions and one mononucleotide repeat (MNR) locus was located in a noncoding region. Multiple alleles were found at the studied SSR loci among 47 isolates from various hosts, including eight additional strains mainly from humans (generous gift from Nestle Company, Table 1), revealing a high level of polymorphism among L. johnsonii strains (Table 2). Two strategies were used to identify the 


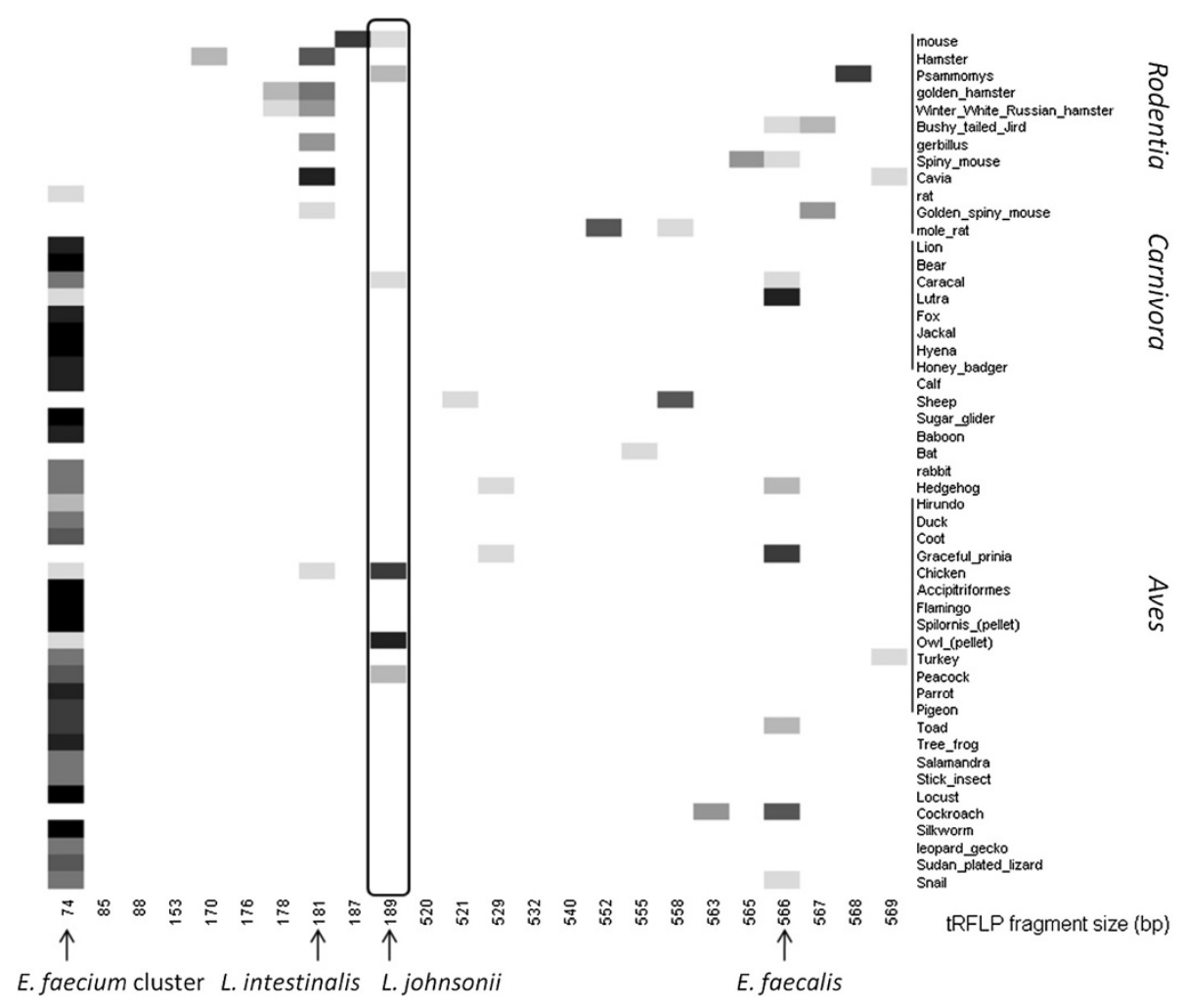

Figure 1 Relative abundances of tRFLP fragments of selected fecal LAB species from $\mathbf{5 0}$ diverse hosts. Selected Lactic acid bacteria (LAB) of representative host individuals were grown on m-Enterococcus agar, where four major bacterial species were identified. Analysis was performed using R software. Shading represents relative abundance, divided into eight levels, with darker shading indicating higher abundance.

polymorphism: sizing for the SSR loci, and sequencing for the MNR locus. Most SSR loci did not amplify any product (a null allele) in some of the isolates (Table 2). Variation at the MNR locus was observed only in the repeated tract, while the flanking sequences were conserved among isolates. All SSR loci presented 2 to 10 alleles with corresponding diversity indices ranging from 0.28 to 0.76 .

\section{Sequence variation at conserved hypothetical genes}

Three conserved hypothetical genes were chosen for MLST (Table 2). Most isolates gave the expected product size, except for nine isolates which had a deletion of $903 \mathrm{bp}$ in the LJ0017 gene. The Psammomys isolate (LJ_56) did not amplify any product in any of the genes. Sequence variation among isolates was rather high (12.3\%), yielding 236 SNPs out of 1922 bp sequenced in the three genes (Table 2). This variation ranged from 10 to 24 sequence types at a gene, including null alleles, indicating rather high variation among L. johnsonii strains.

\section{Phylogenetic analyses}

The variation data at SSR loci and conserved hypothetical genes were used in two separate analyses to infer the genetic relationships among $L$. johnsonii isolates.
SSR analysis: The phylogenetic analysis divided the $47 \mathrm{~L}$. johnsonii isolates into 29 different SSR types, revealing high discrimination. The resulting dendrogram presented three main clusters (Figure 2A), one composed of chicken and turkey isolates, the second of human isolates and the third of identical mouse isolates together with strains isolated from the caracal feces and the owl pellet (LJ_184, LJ_188, LJ_16 and LJ_252). Note that the owl pellet isolates might be related to the mouse isolates, as it might have originated from the owl's prey (a mouse), rather than from the owl's upper GIT. The isolates from other diverse origins were spread out along the dendrogram. Among them, isolates from Psammomys (LJ_9-7) and silkworm (LJ_4-4), two unrelated host species, are undistinguished according to the typing results. This might be due to their common isolation location, thus additional sampling should clarify the phylogeny clustering of $L$. johnsonii isolates from these two host species. The genetic distances within strains from each of the three groups were significantly low (average genetic distance of $0.25 \pm 0.11,0.27 \pm 0.25$ and $0.11 \pm 0.12$ for chicken, human and mouse clusters, respectively) compared to the high genetic distances observed between isolates from the tested group and the remaining isolates 
Table 1 L. johnsonii strains isolated from feces samples originated in various animals

\begin{tabular}{|c|c|}
\hline Isolate name & Origin \\
\hline L__313, LJ_320 & Domestic chicken line (laying hen) \\
\hline LJ_323 & Domestic chicken line (local line) \\
\hline LJ_363, LJ_365, LJ_368, LJ_c1-2, LJ_c2-6, LJ_c2-8, L_c4-6, LJ_c5-1 & Domestic chicken line (broiler, Ross strain) from two different farms \\
\hline LJ_c3-6, LJ_c3-4 & Domestic chicken line (broiler, Cobb strain) \\
\hline L_ch1, LJ_332, LJ_337 & Domestic chicken line (White Rock broiler) \\
\hline LJ_c6-5, LJ_352, LJ_353 & Turkey \\
\hline LJ_16, LJ31, LJ_32, LJ_36 & Mice (line $\mathrm{C} 57 \mathrm{BL} / 6 \mathrm{~J})$, from two different breeders \\
\hline LJ_5, LJ_10, L__16-1, LJ_18 & Mice (line BALB/C) \\
\hline L_11-2 & Winter white Russian hamster \\
\hline L」_12-5 & Golden hamster \\
\hline L」_15-8 & Rat \\
\hline LJ_56, LJ_9-7 & Psammomys (a type of rat) \\
\hline LJ_184, LJ_188 & Owl (Bubo; sample was obtained from pellet sample) \\
\hline L」_252 & Caracal \\
\hline L_4-4 & Silkworm \\
\hline LJ_Mika1 & Dog \\
\hline L」380 & Calf \\
\hline L」_440 & Peacock \\
\hline NCC 533, NCC 1646, NCC 1657, NCC 1669, NCC 1717, NCC 1741 & Human (Nestle Research Center) \\
\hline NCC 1627 & Unknown source (Nestle Research Center) \\
\hline NCC 1703 & Cheese (Nestle Research Center) \\
\hline
\end{tabular}

Table 2 Number of alleles and diversity index values at the studied 14 loci among $L$. johnsonii isolates

\begin{tabular}{|c|c|c|c|c|}
\hline Locus & Core motif size $(b p)$ and no. of repeats ${ }^{a, b}$ & Gene product & No. of alleles or $\mathrm{ST}^{\mathrm{c}, \mathrm{d}}$ & Diversity index \\
\hline \multicolumn{5}{|l|}{ SSR loci } \\
\hline ᄂJ480 & $(480)_{3}$ & Hypothetical protein & 5 & 0.47 \\
\hline LJ90 & $(90)_{9}$ & Hypothetical protein & 7 & 0.56 \\
\hline LJ66 & $(66)_{7}$ & Hypothetical protein & 5 & 0.50 \\
\hline LJ27 & $(27)_{6}$ & Hypothetical protein & 10 & 0.76 \\
\hline LJ18 & $(18)_{3}$ & Hypothetical protein & 2 & 0.28 \\
\hline LJ12 & $(12)_{4}$ & Signal recognition particle receptor FtsY & 7 & 0.72 \\
\hline LJ9 & $(9)_{3}$ & $\begin{array}{l}\text { Phosphoenolpyruvate-dependent sugar } \\
\text { phosphotransferase system EIIC }\end{array}$ & 3 & 0.66 \\
\hline LJ6 & $(6)_{7}$ & Putative tyrosine-protein kinase & 6 & 0.74 \\
\hline LJ6_1 & $(6)_{3}$ & Cell-wall associated serine proteinase & 3 & 0.29 \\
\hline LJ3 & $(3)_{5}$ & Hypothetical protein & 4 & 0.64 \\
\hline LJ_mono & $(1)_{11}$ & Noncoding & 5 & 0.44 \\
\hline MLST & Sequence length ${ }^{\mathrm{b}}(\mathrm{bp})$ & & & \\
\hline LJ0017 & 1113 & 'Conserved hypothetical' gene & 23 & \\
\hline LJ0648 & 522 & 'Conserved hypothetical' gene & 24 & \\
\hline LJ1632 & 286 & 'Conserved hypothetical' gene & 10 & \\
\hline
\end{tabular}

a Subscript numbers are numbers of motif repeats. SSR loci have non-perfect repeats except for loci LJ3 and $U_{-}$mono.

${ }^{\mathrm{b}}$ Based on the genome sequence of $L$. johnsonii NCC 533.

' Allele: number of repeat variant at SSR; ST: number of sequence types at 'Conserved hypothetical' genes.

${ }^{\mathrm{d}}$ No. of alleles or ST: MLST genes and SSR loci, except for the locus LJ3, included a null allele.

e Isolates: LJ_352, LJ_353, LJ_363, LJ_365, LJ_ch1, LJ_c2-8, LJ_c5-1, Lc_3-4 and L_c6-5 had a deletion of 903 bp. 

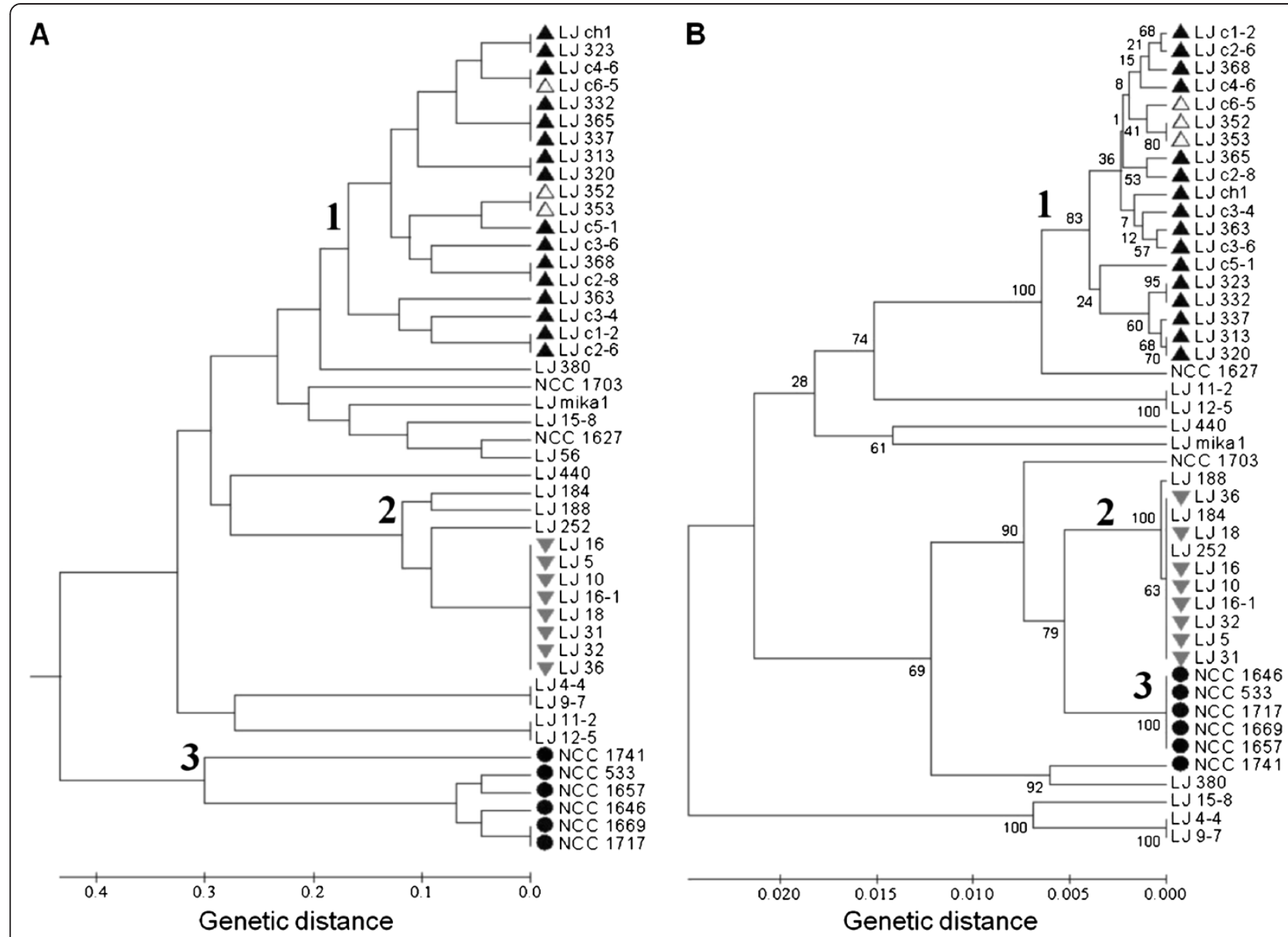

Figure 2 Genetic relationships among L. johnsonii isolates. Dendograms are based on variation data of: (A) 47 isolates at 11 SSR loci based on 57 polymorphic points (11 loci times the number of alleles in each locus); (B) sequence of 46 isolates at three conserved hypothetical genes. Both dendrograms were constructed by UPGMA cluster analysis. Samples from: chickens - $\mathbf{\Lambda}$, turkeys - $\Delta$, humans - $\bullet$ and mice - $\nabla$ are indicated. All the isolation sources of the tested L. johnsonii strains are indicated at Table 1.

(average genetic distance of $0.65 \pm 0.18,0.87 \pm 0.10$ and $0.64 \pm 0.12$ for chicken, human and mouse clusters, respectively).

MLST analysis: phylogenetic analysis of the sequences at the three conserved hypothetical genes separated the 46 typable L. johnsonii isolates into 28 sequence types (Figure 2B). Three clear clusters were obtained, paralleling the SSR analysis, with the exception of strain NCC 1741.

In general, the two genetic analyses similarly separated L. johnsonii isolates into three groups (Figure 2A, 2B). The clusters included strains with a common isolation host: various lines of chicken and turkey, humans, and laboratory mouse lines, while the isolates originating from other diverse sources were dispersed along the dendrograms.

\section{Discussion}

The gut is a habitat for complex bacterial populations, composed of a large variety of bacterial species. Here we concentrated in L. johnsonii, a potentially probiotic bacterial species that is of major interest to the pharmaceutical and food industries as it includes several known probiotic strains [25,28,29]. We successfully identified and isolated $39 \mathrm{~L}$. johnsonii strains from fecal-bacterial populations of few host species.

Strain typing of these isolates together with six additional strains of human origin revealed high levels of genetic variation among the L. johnsonii strains. Both SSR and MLST analyses were found to be effective for typing, providing high-resolution discrimination also among isolates originated in the same animal species. The genetic relationships among the strains inferred by the two analyses were similar, clearly dividing the L. johnsonii strains into three clusters. Each cluster consisted of strains from different diverse hosts, i.e., chickens, humans or mice (Figure 2). These consistent results, obtained by different typing methods, suggest far phylogenetic separation among L. johnsonii isolates 
presenting host specificity. Such association of particular L. johnsonii strains with the host taxonomy could arise as a result of co-evolution of the host and its GIT microbiota [2,41-43]. Interestingly, host driven evolution was observed in another lactobacilli species, L. reuteri [44]. According to the recently suggested "hologenome theory" [45], the host and its symbiont microbiota (together defined as the "holobiont") are one unit of selection in evolution. Indeed, previous analysis of the L. johnsonii genome showed the absence of genes required for several metabolic pathways [29] emphasizing the high dependence of $L$. johnsonii on its host and further supports the concept that $L$. johnsonii and its host are one evolutionary unit of selection. Since chickens, humans and mice are distinct genetic species divided during evolution, L. johnsonii strains associated with them may be evolutionary separated as part of the distinct holobionts.

In addition, analysis conducted on the tRFLP results of 50 host individuals suggest an association of L. intestinalis and E. faecium cluster with host taxonomic groups (Figure 1), and further support co-evolution of the host and its intestinal bacteria. The E. faecium species cluster was relatively rare in hosts belonging to the Rodentia taxonomic order, and alternatively, L. intestinalis was found to be more frequent within that group. These observations may indicate possible competition or a similar function of these two bacteria in the same niche, each within its appropriate microenvironment. Environmental factors, such as diet, are highly important in shaping the host gut's microbiota composition [4-6,46]. However, in our study, no correlation was found between the presence of each of the four bacterial species tested and the hosts' food consumption (herbivore, omnivore and carnivore) or geographical location.

\section{Conclusions}

L. johnsonii strain typing conducted here support host specificity of $L$. johnsonii only at the strain level. tRFLP analysis of a narrow spectrum of fecal LAB populations demonstrated host specificity of $L$. intestinalis and the $E$. faecium cluster at the species level of bacteria. Both observations suggest co-evolution of the bacteria, either at the species or the strain level, with distinct animal species.

The identified bacterial host specificity may be further applied to utilization of health-promoting specific strains based on the bacterium and the host's genetics, as part of the personalized medicine approach.

\section{Methods}

\section{Isolation procedure and growth conditions}

A total of 104 samples were collected from a wide variety of animal hosts, originated in 58 animal species. Samples were collected in Israel during a 1.5 year period
(January 2009 - June 2010). 102 samples were feces samples, and 2 were bird pellets, i.e the materials regurgitated by the birds (see Additional file 1: Origin of samples collected from 104 animal hosts). Each sample, obtained from individual host, was treated and analyzed separately. Samples were kept at $4^{\circ} \mathrm{C}$ in $0.1 \mathrm{M}$ sodium phosphate buffer $\mathrm{pH} 7$ until arrival to the lab (up to $4 \mathrm{~h}$ from the collection time) and processed immediately. $0.1 \mathrm{M}$ sodium phosphate buffer $\mathrm{pH} 7$ was added to a final concentration of $10 \%(\mathrm{w} / \mathrm{v})$, to equally normalize the growth of fecal bacteria from all samples (see below) according the feces weight. Samples were homogenized by vigorous vortexing, followed by centrifugation at $1500 \times g$, at $4^{\circ} \mathrm{C}$ for $5 \mathrm{~min}$. The supernatant containing the bacterial suspension was transferred to a clean tube. A $100 \mu \mathrm{l}$ aliquot of bacterial suspension was spread on either MRS agar (de Man, Rogosa, Sharpe; Oxoid, UK) or DIFCO m-Enterococcus agar plates (BD, Maryland, USA), and grown under both aerobic and anaerobic conditions at $37^{\circ} \mathrm{C}$ for $48 \mathrm{~h}$. mEnterococcus agar was used to isolate L. johnsonii based on our previous study [8].

Total DNA was extracted from samples of the bacterial populations grown on the anaerobically incubated $\mathrm{mEnterococcus} \mathrm{agar} \mathrm{plates} \mathrm{and} \mathrm{terminal} \mathrm{restriction} \mathrm{frag-}$ ment length polymorphism (tRFLP) was performed, in order to assess the presence of L. johnsonii within the total bacterial population that grew on the plate. tRFLP was conducted only for plates that presented massive bacterial growth, estimated at few dozen colonies and more (plates from 62 samples). These samples belong to hosts from six taxonomic classes, in which Mammalia (34 samples) and Aves (18 samples) were the most abundant. The mammalian hosts belonged to eight different orders, most from Rodentia (15 samples) and Carnivora (9 samples). Totally, the 62 samples belong to 50 different animal species.

To isolate L. johnsonii, aerobically and anaerobically incubated mEnterococcus and MRS agar plates were screened for L. johnsonii presence, where primary selection was according to slight differences in colony morphology of different species (in comparison to known L. Johnsonii) and based on tRFLP results for the 62 samples. Colonies suspected of being L. johnsonii were picked for PCR amplification with species-specific primers designed to the $23 S$ rDNA (see section Locus and primer selection). Final verification was achieved by $16 S$ rDNA sequencing [GenBank: JN 012220 - JN 012227 for $16 S$ rDNA sequences of LJ56, LJ313, LJ363, LJ380, LJc1-2, LJc3-4, LJc3-6 and LJmika1, respectively. The $16 S$ rDNA sequences of the other $L$. johnsonii isolates are similar to the sequence of LJ16, GenBank: JF923644]. $16 S \mathrm{rDNA}$ sequences of colonies with slightly different morphologies were indeed proven not to be L. johnsonii. 
Pure L. johnsonii cultures were grown in MRS broth (de Man, Rogosa, Sharpe; Oxoid, UK) overnight at $37^{\circ} \mathrm{C}$, freeze-dried and kept at $-20^{\circ} \mathrm{C}$ in the presence of trehalose and maltodextrin, as previously described [47].

\section{DNA extraction}

Cells were harvested from either a loop full of fecalbacterial population grown on $\mathrm{mEnterococcus}$ agar plates or pure overnight culture of L. johnsonii $(200 \mu \mathrm{l})$ grown in MRS broth that was centrifuged at $12,000 \times g$ for $1 \mathrm{~min}$. Cells were suspended in $1 \mathrm{ml}$ of $70 \%$ ethanol by vigorous vortexing, $33 \mu \mathrm{l}$ of $3 \mathrm{M}$ sodium acetate ( $\mathrm{pH}$ 5.2) was added and the samples were incubated at $-80^{\circ} \mathrm{C}$ for $20 \mathrm{~min}$, followed by centrifugation at $12,000 \times g$ for $15 \mathrm{~min}$. The supernatant was decanted and the pellet was dissolved in $30 \mu \mathrm{l}$ of $0.1 \times$ Tris-EDTA buffer (TE). The crude DNA was diluted 10 -fold and stored at $-20^{\circ} \mathrm{C}$.

\section{tRFLP of fecal-bacterial population}

$16 S r D N A$ of the fecal-bacterial population was amplified in a total volume of $50 \mu \mathrm{l}$ using 27 F-FAM fluorophorelabeled primer and 1492R primer [48] together with $10 \mu \mathrm{l}$ of 1:10-diluted crude DNA, at an annealing temperature of $60^{\circ} \mathrm{C}$ (see section PCR and Additional file 2: Primers and their annealing temperatures (Tm)). The PCR products were purified by ethanol precipitation and dissolved in $20 \mu \mathrm{lddH_{2 }}$ O. A 1- $\mu$ g aliquot of the purified PCR product was digested with $20 \mathrm{U} M s p 1$ restriction enzyme (New England Biolabs) in a total volume of $20 \mu \mathrm{l}$ for $2 \mathrm{~h} 15 \mathrm{~min}$ at $37^{\circ} \mathrm{C}$ followed by enzyme inactivation at $65^{\circ} \mathrm{C}$ for $20 \mathrm{~min}$. A 50-ng aliquot of the digested DNA was loaded into an ABI 3130 genetic analyzer together with $9 \mu$ formamide and $0.5 \mu$ l GeneScan 1200 LIZ size standard (Applied Biosystems, California, USA) for size determination. The results were analyzed using GeneMapper 4.0 software (Applied Biosystems).

The species identification of an isolated bacterial colony was performed by terminal restriction fragment analysis followed by $16 \mathrm{~S}$ rDNA sequencing and by in silico t-RFLP analysis for verification (http://insilico.ehu.es/ T-RFLP/, [49]).

The GenBank nucleotide accession numbers of the identified species representing the tRFLP peaks of 74, 181, 189 and 566 bp (Figure 1, Additional file 3: tRFLP patterns of a selected fecal LAB populations obtained from three representative animal hosts) are: Enterococcus faecium species cluster [GenBank: JF923641, JF923642], Lactobacillus intestinalis [GenBank: JF923643], L. johnsonii [GenBank: JF923644], and Enterococcus faecalis [GenBank: JF923645].

\section{Screening of the genome for SSR distribution}

The complete genomic sequence of L. johnsonii NCC 533, obtained from the NCBI database, was screened for perfect SSR (i.e., exact-repeat motifs) using the "SSR" computer program [37,50], and for non-perfect SSR (NP-SSR, i.e. non-exact repeat motifs) using the "ATR Hunter" computer program (http://bioinfo.cs.technion. ac.il/atrhunter/ATRHunter.htm [51]). Perfect SSR included mononucleotide repeats (MNR) with longer than 5-bp repeats, and large SSR with motif size $\geq 3 \mathrm{bp}$ repeated more than twice. NP-SSR included only SSR with motif size $\geq 3$ bp and minimal similarity between repeats of more than $70 \%$.

\section{Locus and primer selection}

SSR loci: Eleven loci (Additional file 2: Primers and their annealing temperatures ( $\mathrm{Tm})$ ) were chosen for the study, including ten SSR loci and one MNR locus. These regions exhibited no similarity to phage or prophage sequences. Unique primers were designed to generate PCR products of 120 to $1650 \mathrm{bp}$ using the Gene Runner software (version 3.05; Hastings Software Inc.). Each locus was tested for uniqueness in the $L$. johnsonii genome by using NCBI BLAST (http://www.ncbi.nlm.nih. gov/sutils/genom_table.cgi).

Species-specific primers: L. johnsonii-specific primers were designed based on the $23 S \mathrm{rDNA}$ sequences of a variety of lactobacilli available at the NCBI database. The forward primer was designed such that the last nucleotide at the 3' end of the primer was unique to $L$. johnsonii. The reverse primer was designed based on a previously designed L. johnsonii-specific probe [52]. Species-specific PCR amplification $\left(\mathrm{Tm}=51^{\circ} \mathrm{C}\right.$, Additional file 2: Primers and their annealing temperatures (Tm)) was performed directly on the colonies of the suspected $L$. johnsonii isolates.

Conserved hypothetical genes: Three conserved hypothetical genes were chosen for the MLST from the JCVI CMR database (http://cmr.jcvi.org/cgi-bin/CMR/ CmrHomePage.cgi) based on the genome sequence of L. johnsonii NCC 533. Gene choice was based on two criteria: (i) presence in other L. johnsonii strains, and (ii) a high number of single nucleotide polymorphisms (SNPs) compared to the sequence of L. johnsonii ATCC 32000 in the NCBI database. Unique primers were designed to generate PCR products of 400 to 1200 bp (Additional file 2: Primers and their annealing temperatures (Tm)). Due to the non-amplification of products in a few strains, additional primer sets were designed for each of the genes (LJ0017_new, LJ_0648_new and LJ_1632_new) based on the sequences obtained for the rest of the isolates.

\section{PCR}

Each PCR mixture contained $0.2 \mathrm{mM}$ deoxynucleoside triphosphates, $0.4 \mu \mathrm{M}$ forward and reverse primers, 0.02 $\mathrm{U} / \mu \mathrm{l}$ of Taq polymerase (SuperNova, JMR Holding, Kent, England), $1 \times$ reaction buffer (containing $1.5 \mathrm{mM}$ 
$\mathrm{MgCl}_{2}$ ) and $5 \mu \mathrm{l}$ of 1:10-diluted crude DNA in a total volume of $25 \mu \mathrm{l}$. The reactions were carried out in a Veriti 96-well thermal cycler (Applied Biosystems, California, USA) as follows: $95^{\circ} \mathrm{C}$ for $3 \mathrm{~min} ; 30$ cycles of $30 \mathrm{~s}$ at $95^{\circ} \mathrm{C}, 30 \mathrm{~s}$ at the annealing temperature (Tm, Additional file 2: Primers and their annealing temperatures $(\mathrm{Tm})$ ), and $90 \mathrm{~s}$ at $72^{\circ} \mathrm{C} ; 10 \mathrm{~min}$ at $72^{\circ} \mathrm{C}$, and cooling to $12^{\circ} \mathrm{C}$. PCR products were verified by gel (1.2\%) electrophoresis and observed by UV fluorescence.

\section{DNA sizing}

Size determination of SSR amplification products with motif lengths of $66 \mathrm{bp}, 90 \mathrm{bp}$ and $480 \mathrm{bp}$ was performed by $2 \%$ agarose gel electrophoresis. Sizing of the other seven SSR loci was performed by capillary electrophoresis on an ABI 3130 genetic analyzer, using fluorophorelabeled primers. The amplification products were loaded into the genetic analyzer together with $9 \mu$ formamide and $0.5 \mu \mathrm{l} \mathrm{GeneScan} 500 \mathrm{LIZ}$ size standard (Applied Biosystems). The results were analyzed with GeneMapper 4.0 software (Applied Biosystems).

\section{DNA sequencing}

PCR amplification products were purified using a QIAquick PCR purification kit (Qiagen, Hilden, Germany). Purified DNA (20-50 ng) was sequenced on both strands using a BigDye terminator v1.1 cycle sequencing kit (Applied Biosystems) and loaded into the ABI 3130 genetic analyzer. Results were analyzed with SeqScape 2.5 software (Applied Biosystems) and DNA sequencing analysis 5.2 software (Applied Biosystems).

GenBank numbers of nucleotide sequences for genes LJ_0017, LJ_0648 and LJ_1632: JN012103 - JN 012141, JN 012142 - JN 012180 and JN 012181 - JN 012219 respectively.

\section{Data and statistical analyses}

tRFLP: The relative abundance of each tRFLP peak was calculated as the peak area divided by the total area summed over all peaks in a sample. A statistical analysis was performed for each of the four main tRFLP peaks (74 bp, $181 \mathrm{bp}, 189 \mathrm{bp}$ and $566 \mathrm{bp}$ ) separately. M-ANOVA (JMP 8.0) was performed based on the relative abundance of each tested peak in each sample to compare its presence among the 50 tested samples under three parameters (geographical location, taxonomy and food classification). The software $\mathrm{R}$ was used to present the relative abundances of the tRFLP patterns, split into eight levels.

Sequence comparison: The obtained $16 \quad S$ rDNA sequences were compared to all available sequences using the NCBI BLAST algorithm for species identification. The analysis of the sequence variation data was performed on the combined sequences of the three conserved hypothetical genes for each of the 46 strains. One strain (LJ_56) did not give any amplification product and was therefore excluded from the MLST analysis. Multiple sequence alignments were performed using CLUSTALW software [53]. The alignment files were converted to MEGA format and used to evaluate genetic relationships among the strains by the unweighted pair group method with arithmetic mean (UPGMA) (MEGA 4.0 [54]).

Allele analysis: A nonparametric analysis of allelic variation was used for all $47 \mathrm{~L}$. johnsonii isolates at SSR loci, with alleles referring to SSR size alleles. For the MNR locus, alleles referred to the MNR size, similar to the SSR loci, as no sequence variation was obtained in the flanking regions of the MNR. An additional allele was counted where there was no amplification product. The data for all genotypes were scored as present (" 1 ") or absent ("0") for each allele at a specific locus. Diversity index was calculated as 1 $\sum \mathrm{P}_{i j}^{2}$, where $\mathrm{P}_{i j}$ is the frequency of the $j$ th allele at the $i$ th locus. Genetic relationships were inferred among strains based on the variation data. SAS software was used to calculate the Nei coefficient of association and to generate the corresponding matrix (SAS system for Windows, version 9.02; SAS Institute, Inc., Cary, NC). The matrix was used to create dendograms based on the UPGMA using MEGA 4.0 software [54]. Bootstrap confidence values were based on 1,000 simulated dendrograms.

\section{Additional files}

Additional file 1: Origin of samples collected from 104 animal hosts.

Additional file 2: Primers and their annealing temperatures.

Additional file 3: tRFLP patterns of selected fecal LAB populations obtained from three representative animal hosts. Bacteria were grown on m-Enterococcus agar. Fluorescent-labeled DNA fragments were analyzed by $A B I 3130$ genetic analyzer. The size of specific fragments is indicated in bp. The owl sample is a pellet sample.

\section{Authors' contributions}

$K B, Y D, H S$, YK conceived and designed the study. KB, VM and MJ carried out the experiments. $K B$ and $Y D$ analyzed results. $K B, Y D$ and $Y K$ drafted the manuscript. All authors read and approved the final manuscript.

\section{Acknowledgements}

We are grateful to Nestec Company (Nestec Ltd., Nestle Research Center Lausanne, P.O. Box 44, CH-1000 Lausanne 26) for providing L. johnsonii strains, as well as to Haifa zoo, Haifa university (Oranim campus), and Hayogev and Ramat Yohanan framers for their co-operation in the feces sampling.

\section{Author details}

${ }^{1}$ Department of Biotechnology and Food Engineering, Technion-Israel Institute of Technology, Haifa, Israel. ${ }^{2}$ School of Life Sciences, Handong Global University, Pohang, Gyungbuk 791-708, Korea.

Received: 29 December 2011 Accepted: 29 June 2012 Published: 25 July 2012

\section{References}

1. Costello EK, Lauber CL, Hamady M, Fierer N, Gordon Jl, Knight R: Bacterial community variation in human body habitats across space and time. Science 2009, 326:1694-1697. 
2. Dethlefsen L, McFall-Ngai M, Relman DA: An ecological and evolutionary perspective on human-microbe mutualism and disease. Nature 2007, 449:811-818.

3. Eckburg PB, Bik EM, Bernstein CN, Purdom E, Dethlefsen L, Sargent M, Gill $\mathrm{SR}$, Nelson KE, Relman DA: Diversity of the human intestinal microbial flora. Science 2005, 308:1635-1638.

4. Ley RE, Hamady M, Lozupone C, Turnbaugh PJ, Ramey RR, Bircher JS, Schlegel ML, Tucker TA, Schrenzel MD, Knight R, et al: Evolution of mammals and their gut microbes. Science 2008, 320:1647-1651.

5. Mshvildadze M, Neu J, Mai V: Intestinal microbiota development in the premature neonate: establishment of a lasting commensal relationship? Nutr Rev 2008, 66:658-663.

6. Turnbaugh PJ, Ridaura VK, Faith JJ, Rey FE, Knight R, Gordon Jl: The effect of diet on the human gut microbiome: a metagenomic analysis in humanized gnotobiotic mice. Sci Trans/ Med 2009, 1:6-14

7. Benson AK, Kelly SA, Legge R, Ma F, Low SJ, Kim J, Zhang M, Oh PL, Nehrenberg D, Hua K, et al: Individuality in gut microbiota composition is a complex polygenic trait shaped by multiple environmental and host genetic factors. Proc Natl Acad Sci USA 2010, 107:18933-18938.

8. Buhnik-Rosenblau K, Danin-Poleg Y, Kashi Y: Predominant Effect of Host Genetics on Levels of Lactobacillus johnsonii bacteria in the Mouse Gut. Appl Environ Microbiol 2011, in press.

9. Khachatryan ZA, Ktsoyan ZA, Manukyan GP, Kelly D, Ghazaryan KA, Aminov Rl: Predominant Role of Host Genetics in Controlling the Composition of Gut Microbiota. PLoS One 2008, 3:e3064.

10. Spor A, Koren O, Ley R: Unravelling the effects of the environment and host genotype on the gut microbiome. Nat Rev Microbiol 2011, 9:279-290.

11. Zoetendal EG, Akkermans ADL, Akkermans-van Vliet WM, de Visser JAGM, de Vos WM: The host genotype affects the bacterial community in the human gastrointestinal tract. Microbial ecol Health Dis 2001, 13:129-134.

12. Haller D, Antoine JM, Bengmark S, Erick P, Rijkers GT, Lenoir-Wijnkoop I: Guidance for Substantiating the Evidence for Beneficial Effects of Probiotics: Probiotics in Chronic Inflammatory Bowel Disease and the Functional Disorder Irritable Bowel Syndrome. J Nutr 2010, 140:690S-697S.

13. Holubar SD, Cima RR, Sandborn WJ, Pardi DS: Treatment and prevention of pouchitis after ileal pouch-anal anastomosis for chronic ulcerative colitis. Cochrane Database Syst Rev 2010, 6:CD001176.

14. Kalliomaki M, Antoine JM, Herz U, Rijkers GT, Wells JM, Mercenier A: Guidance for Substantiating the Evidence for Beneficial Effects of Probiotics: Prevention and Management of Allergic Diseases by Probiotics. J Nutr 2010, 140:713S-721S.

15. Lionetti E, Indrio F, Pavone L, Borrelli G, Cavallo L, Francavilla R: Role of probiotics in pediatric patients with Helicobacter pylori infection: a comprehensive review of the literature. Helicobacter 2010, 15:79-87.

16. Wolvers D, Antoine JM, Myllyluoma E, Schrezenmeir J, Szajewska H, Rijkers GT: Guidance for Substantiating the Evidence for Beneficial Effects of Probiotics: Prevention and Management of Infections by Probiotics. J Nutr 2010, 140:698S-712S.

17. Claesson MJ, van Sinderen D, OToole PW: The genus Lactobacillus - a genomic basis for understanding its diversity. FEMS Microbiol Lett 2007, 269:22-28.

18. Felis EF, Dellaglio F: Taxonomy of Lactobacilli and Bifidobacteria. Curr Issues Intest Microbiol 2007

19. Kim SY, Adachi Y: Biological and genetic classification of canine intestinal lactic acid bacteria and bifidobacteria. Microbiol Immunol 2007, 51:919-928

20. Pena JA, Li SY, Wilson PH, Thibodeau SA, Szary AJ, Versalovic J: Genotypic and phenotypic studies of murine intestinal lactobacilli: Species differences in mice with and without colitis. Appl Environ Microbiol 2004, 70:558-568

21. Stephenson DP, Moore RJ, Allison GE: Comparison and Utilization of Repetitive-Element PCR Techniques for Typing Lactobacillus Isolates from the Chicken Gastrointestinal Tract. Appl Environ Microbiol 2009, 75:6764-6776.

22. Audisio MC, Torres MJ, Sabate DC, Ibarguren C, Apella MC: Properties of different lactic acid bacteria isolated from Apis mellifera L. bee-gut. Microbiol Res 2011, 166:1-13.

23. Korhonen JM, Sclivagnotis Y, von Wright A: Characterization of dominant cultivable lactobacilli and their antibiotic resistance profiles from faecal samples of weaning piglets. J Appl Microbiol 2007, 103:2496-2503.
24. Lai KK, Lorca GL, Gonzalez CF: Biochemical Properties of Two Cinnamoyl Esterases Purified from a Lactobacillus johnsonii Strain Isolated from Stool Samples of Diabetes-Resistant Rats. Appl Environ Microbiol 2009, 75:5018-5024.

25. Van Coillie E, Goris J, Cleenwerck I, Grijspeerdt K, Botteldoorn N, Van Immerseel F, De Buck J, Vancanneyt M, Swings J, Herman L, et al: Identification of lactobacilli isolated from the cloaca and vagina of laying hens and characterization for potential use as probiotics to control Salmonella Enteritidis. J Appl Microbiol 2007, 102:1095-1106.

26. Pinto MGV, Schuster T, Briviba K, Watzl B, Holzapfel WH, Franz CMAP. Adhesive and chemokine stimulatory properties of potentially probiotic Lactobacillus strains. J Food Protection 2007, 70:125-134.

27. du Toit M, Franz CMAP, Dicks LMT, Schillinger U, Haberer P, Warlies B, Ahrens F, Holzapfel WH: Characterisation and selection of probiotic lactobacilli for a preliminary minipig feeding trial and their effect on serum cholesterol levels, faeces $\mathrm{pH}$ and faeces moisture content. Int $J$ Food Microbiol 1998, 40:93-104.

28. La Ragione RM, Narbad A, Gasson MJ, Woodward MJ: In vivo characterization of Lactobacillus johnsonii FI9785 for use as a defined competitive exclusion agent against bacterial pathogens in poultry. Lett Appl Microbiol 2004, 38:197-205.

29. Pridmore RD, Berger B, Desiere F, Vilanova D, Barretto C, Pittet AC, Zwahlen MC, Rouvet M, Altermann E, Barrangou R, et al: The genome sequence of the probiotic intestinal bacterium Lactobacillus johnsonii NCC 533. Proc Nat Acad Sci U S A 2004, 101:2512-2517.

30. Berger B, Pridmore RD, Barretto C, Delmas-Julien F, Schreiber K, Arigoni F, Brussow H: Similarity and differences in the Lactobacillus acidophilus group identified by polyphasic analysis and comparative genomics. J Bacteriol 2007, 189:1311-1321.

31. Guan LL, Hagen KE, Tannock GW, Korver DR, Fasenko GM, Allison GE: Detection and identification of Lactobacillus species in crops of broilers of different ages by using PCR-denaturing gradient gel electrophoresis and amplified ribosomal DNA restriction analysis. Appl Environ Microbiol 2003, 69:6750-6757.

32. Kullen MJ, Sanozky-Dawes RB, Crowell DC, Klaenhammer TR: Use of the DNA sequence of variable regions of the $16 \mathrm{~S}$ rRNA gene for rapid and accurate identification of bacteria in the Lactobacillus acidophilus complex. J Appl Microbiol 2000, 89:511-516.

33. Ventura M, Zink R: Specific identification and molecular typing analysis of Lactobacillus johnsonii by using PCR-based methods and pulsed-field gel electrophoresis. FEMS Microbiol Lett 2002, 217:141-154.

34. Van Ert MN, Easterday WR, Huynh LY, Okinaka RT, Hugh-Jones ME, Ravel J, Zanecki SR, Pearson T, Simonson TS, U'Ren JM, et al: Global Genetic Population Structure of Bacillus anthracis. PLoS One 2007, 2:e461.

35. Danin-Poleg Y, Cohen LA, Gancz H, Broza YY, Goldshmidt H, Malul E, Valinsky L, Lerner L, Broza M, Kashi Y: Vibrio cholerae strain typing and phylogeny study based on simple sequence repeats. J Clin Microbiol 2007, 45:736-746.

36. van Belkum A, Scherer S, van Alphen L, Verbrugh H: Short-sequence DNA repeats in prokaryotic genomes. Microbiol Mol Biol Rev 1998, 62:275-293.

37. Mee-Marquet N, Francois P, Domelier AS, Arnault L, Girard N, Schrenzel J, Quentin R: Variable-Number Tandem Repeat Analysis and Multilocus Sequence Typing Data Confirm the Epidemiological Changes Observed with Staphylococcus aureus Strains Isolated from Bloodstream Infections. J Clin Microbiol 2009, 47:2863-2871.

38. Urwin R, Maiden MCJ: Multi-locus sequence typing: a tool for global epidemiology. Trends Microbiol 2003, 11:479-487.

39. Zhang LQ, Li WH: Mammalian housekeeping genes evolve more slowly than tissue-specific genes. Mol Biol Evol 2004, 21:236-239.

40. Galperin MY, Koonin EV: 'Conserved hypothetical' proteins: prioritization of targets for experimental study. Nucleic Acids Res 2004, 32:5452-5463.

41. Ley RE, Peterson DA, Gordon Jl: Ecological and evolutionary forces shaping microbial diversity in the human intestine. Cell 2006, 124:837-848.

42. McFall-Ngai M: Adaptive immunity - Care for the community. Nature 2007, 445:153.

43. Neish AS: Microbes in Gastrointestinal Health and Disease. Gastroenterology 2009, 136:65-80

44. Oh PL, Benson AK, Peterson DA, Patil PB, Moriyama EN, Roos S, Walter J: Diversification of the gut symbiont Lactobacillus reuteri as a result of host-driven evolution. ISME J 2010, 4:377-387. 
45. Zilber-Rosenberg I, Rosenberg E: Role of microorganisms in the evolution of animals and plants: the hologenome theory of evolution. FEMS Microbiol Rev 2008, 32:723-735.

46. Palmer C, Bik EM, DiGiulio DB, Relman DA, Brown PO: Development of the human infant intestinal microbiota. PLoS Biol 2007, 5:1556-1573.

47. Semyonov D, Ramon O, Kaplun Z, levin-Brener L, Gurevich N, Shimoni E: Food Res Int 2009, 43:193-202.

48. Sakamoto $M$, Hayashi $H$, benno $Y$ : Terminal restriction fragment length polymorphism analysis for human fecal microbiota and its application for analysis of complex bifidobacterial communities. Microbiol Immunol 2002, 47:133-142.

49. Bikandi J, San Millan R, Rementeria A, Garaizar J: In silico analysis of complete bacterial genomes: PCR, AFLP-PCR and endonuclease restriction. Bioinformatics 2004, 20:798-799.

50. Gur-Arie R, Cohen CJ, Eitan Y, Shelef L, Hallerman EM, Kashi Y: Simple sequence repeats in Escherichia coli: Abundance, distribution, composition, and polymorphism. Genome Res 2000, 10:62-71.

51. Wexler Y, Yakhini Z, Kashi Y, Geiger D: Finding approximate tandem repeats in genomic sequences. J Comput Biol 2005, 12:928-942.

52. Park SH, Itoh K: Species-specific oligonucleotide probes for the detection and identification of Lactobacillus isolated from mouse faeces. J Appl Microbiol 2005, 99:51-57.

53. Thompson JD, Higgins DG, Gibson TJ: Clustal-W - Improving the Sensitivity of Progressive Multiple Sequence Alignment Through Sequence Weighting, Position-Specific Gap Penalties and Weight Matrix Choice. Nucleic Acids Res 1994, 22:4673-4680.

54. Tamura K, Dudley J, Nei M, Kumar S: MEGA4: Molecular Evolutionary Genetics Analysis (MEGA) software version 4.0. Mol Biol Evol 2007, 24:1596-1599.

doi:10.1186/1471-2180-12-149

Cite this article as: Buhnik-Rosenblau et al: Indication for Co-evolution of Lactobacillus johnsonii with its hosts. BMC Microbiology 2012 12:149.

\section{Submit your next manuscript to BioMed Central and take full advantage of:}

- Convenient online submission

- Thorough peer review

- No space constraints or color figure charges

- Immediate publication on acceptance

- Inclusion in PubMed, CAS, Scopus and Google Scholar

- Research which is freely available for redistribution 